\title{
Report of the Tobacco Policy Research Study Group on Smoke-Free Indoor Air Policies
}

\author{
David M Burns, Robert Axelrad, Dileep Bal, Julia Carol, Ronald M Davis, \\ Matthew L Myers, John M Pinney, Nancy A Rigotti, Donald R Shopland
}

University of California, San Diego, UCSD Medical Center, San Diego,

\section{California}

D M Burns

Environmental

Protection Agency,

Washington, DC

R Axelrad

California State

Department of

Health, Los Angeles,

California

D Bal

Americans for

Nonsmokers' Rights,

Berkeley, California

J Carol

Michigan Department of Public Health, Lansing, Michigan

R M Davis

Asbill, Junkin, and Myers, Chtd, and the Coalition on Smoking or Health,

Washington, DC

M L Myers

Corporate Health

Policies Group,

Washington, DC

J M Pinney

Harvard Medical School, Boston

Massachusetts

N A Rigotti

National Cancer

Institute, Bethesda,

Maryland

D R Shopland

Correspondence to: $\mathrm{Dr}$ David M Burns, University of California, UCSD Medical Center, 225 Dickenson Street, San Diego, California 921021990, USA.
The rationale for policies that restrict the locations where smoking is permitted reflects three major issues:

- Environmental tobacco smoke (ETS) is annoying and irritating to most non-smokers

- ETS can cause acute and chronic illness in non-smokers

- Restricting the locations where smoking is allowed may increase the number of smokers who attempt to stop smoking; may reduce the frequency of relapse by those who attempt to stop; and may reduce the number of cigarettes smoked by those who continue to smoke.

This paper reviews the evidence that ETS requires restrictive public policies, and discusses the various mechanisms used to accomplish this. It provides background on the many laws, regulations, and ordinances implemented in the United States to date and draws attention to issues requiring further research to strengthen public policy in this area.

\section{Background}

The existing research base clearly establishes that many non-smokers are annoyed and physically irritated by exposure to ETS at levels that occur when smoking is allowed. 'It is also clear that ETS produces acute and chronic illnesses, most notably lung cancer and heart disease in adults ${ }^{2,3}$ and respiratory illness in children. ${ }^{4}$ The precise magnitude of these effects and the range of diseases that are produced by exposure to ETS are issues of current scientific interest. For purposes of public policy, however, there is conclusive scientific proof that both short term and long term exposure to ETS produce significant adverse health outcomes at levels that substantially exceed those of other agents currently restricted and regulated in the United States and several other countries.

Further research on health outcomes will be important in defining the mechanisms by which tobacco smoke causes disease and the extent of the disease burden, but it is not required to define or support the need for public health policy in this area.

There is now a consensus in the scientific community regarding the health impact of ETS. Broadly based reviews of the scientific data in this area by the National Academy of Sciences, ${ }^{5}$ the US Public Health Service, ${ }^{6}$ the Environmental Protection Agency, ${ }^{7}$ and the National Institute for Occupational Safety and
Health $^{8}$ have separately concluded that ETS causes lung cancer and other illnesses in nonsmokers at the levels of exposure that commonly occur in work and home environments in the United States. Most recently, the American Heart Association's council on Cardiopulmonary and Critical Care concluded that ETS is a major preventable cause of cardiovascular disease and death. ${ }^{9}$

Environmental measures of ETS and biological markers of exposure to ETS document that a large proportion of non-smokers is exposed to ETS in a wide variety of environments including worksites. Measures of intensity and variability of exposure across time and location are less common and result in some uncertainty about the magnitude of current and past exposures to ETS for the population of the United States. Further data on these questions could allow more precise estimation of the magnitude of the disease burden caused by exposure to ETS (for example, estimations of the number of lung cancers occurring each year attributable to exposure to ETS), but would not alter the judgement that the disease burden requires protective public health policy.

Mitigating the risk from exposure to ETS through structural alterations in indoor environments and by alterations in air conditioning systems has been investigated. The particle size of the ETS aerosol and its chemical makeup leads to rapid dispersion of ETS throughout the entire airspace in which smoking takes place, including any airspace that is part of a common ventilation system. Air filtration and cleaning systems capable of removing smoke from the air passing through the system are too expensive to be used in most work environments, and can do little to alter the exposures to ETS that occur due to close unobstructed proximity to the smoker. Where smoking is permitted it is possible to reduce, but not eliminate, exposure to ETS by increasing the flow of outdoor air into the indoor environment. For this reason, banning smoking - the source of ETS - is an increasingly common strategy to eliminate the risk of exposure to ETS in worksites and other indoor environments.

Data on the prevalence of regulations and policies that restrict smoking in worksites and other environments show that these policies are becoming both more common and more restrictive. ${ }^{10}$ Support of policies that restrict 
smoking is widespread among non-smokers ${ }^{11}$ and implementation of restrictions in worksites and other public places has been accomplished without significant conflict or expense. ${ }^{12}$ Smokers are highly supportive of policies restricting smoking when surveyed after a policy change has been effectively implemented. Smokers are generally compliant with policies that restrict smoking and these policies are largely self enforcing, with the result that there is a large reduction in the amount of smoking that occurs in a location once smoking is prohibited.

Few data exist on the relative effectiveness of policies with different levels of restrictiveness in reducing the actual exposure of nonsmokers. Data on the fraction of the population that work in environments that have restrictions of different types and data on the fraction of the population actually exposed to ETS (as opposed to covered by a policy) are limited.

The factors that facilitate the implementation of a smoking policy at a worksite have not been studied in a traditional controlled research setting. Ample observational data suggest, however, that phased implementation, consultation with those affected by the policy, and offers of assistance with cessation increase acceptability and improve implementation. Our understanding of implementation of policies has been derived primarily from large worksites and there is less understanding of the characteristics of effective policy design and implementation for small worksites.

The process of developing and passing local ordinances is also well defined from observational data; model ordinances are currently available. Model ordinances require regular revision to keep pace with the rapidly changing public perception of appropriate and acceptable restrictions on smoking behaviour. Data on the time course and extent of compliance with an ordinance after its enactment are limited, as is information on strategies that can be used to facilitate compliance. Few data exist on the effectiveness of different types of ordinances in actually reducing the exposure of non-smokers to ETS. The variability of compliance with different components of an ordinance and the impact of different types of ordinances (for example separate sections $v$ complete bans) on compliance are poorly understood.

The impact of workplace restrictions on the behaviour of smokers has been examined. Smokers smoke fewer cigarettes at work and over a 24 hour period when smoking is banned at the workplace. ${ }^{13}$ Implementing a smoking ban together with an employer supported assistance with cessation results in an attempt to stop by a substantial proportion of smokers. ${ }^{14}$ The effect of a change in smoking policy alone on cessation is less clear. Studies done primarily in health care settings have shown conflicting results on the effect of banning smoking in the worksite on long term cessation of smoking. ${ }^{15}$ The effect of a smoking ban at the worksite on rates of initiation of smoking among workers entering the work- force and on rates of relapse after cessation of smoking is largely unknown.

Further questions include whether smokers and non-smokers preferentially choose worksites based on their smoking restrictions and whether employers who have banned smoking preferentially hire or promote non-smokers.

\section{Areas of research need}

A broad range of important issues are currently under investigation that relate to ETS. This discussion presents only those research questions that would have an important impact on changing public policy.

\section{TRACKING CHANGE}

A substantial momentum currently exists for changing public policy relating to ETS. As a result, both the midpoint and leading edge of policy change are moving rapidly. These changes are both responses to and causes of changing attitudes, beliefs, and behaviours of smokers and non-smokers. Policy changes that are too far ahead of public opinion may fail and policy changes that lag behind may accomplish less than more restrictive policies.

Tracking changes in attitudes, beliefs, and behaviours of non-smokers (for example, asking smokers not to smoke) and smokers (for example, refraining from smoking around others) nationally and regionally would facilitate policy design. It will also be important to track the policies that exist in different sizes and types of businesses, to provide that information to other businesses considering policy changes. To be used most effectively, this information must be rapidly analysed and readily available.

Work is needed to better define the frequency of exposure to ETS in worksites and other locations, and to link these exposures more precisely to the workers involved. This will allow policy changes and strategies to be customised and given better priorities. Locations such as universities and child day care centres are of particular interest. Identifying the locations where exposures continue to occur, despite the presence of policies and ordinances, will help to define the gaps and deficiencies of existing policies and ordinances.

Assessing the effectiveness of different policies (for example, bans $v$ separate sections) can provide data on the need for more restrictive policies and can be used to monitor the effectiveness of these ordinances and policies. Similarly, it is important to track the fraction of the population that is covered by various components of ordinances and policies.

This targeting can be accomplished through a variety of mechanisms, including population and site specific surveys of attitudes, beliefs, and behaviours as well as self reported exposure to ETS and policy coverage. This information should be collected, analysed, and disseminated regularly at intervals of no more than two years.

Collection and analyses of the ordinances 
covering defined geographic areas can be used to assess the extent of coverage of the population.

LAWS, REGULATIONS, AND ORDINANCES

The passage of laws, regulations, or ordinances (hereafter collectively referred to as ordinances) to restrict smoking does not automatically ensure compliance, and the compliance that does occur develops over time. Currently, the mass media informs much of the public about new ordinances and public pressure induces compliance. As passage of ordinances becomes more common, the newsworthiness and press coverage of their passage may diminish. How will this effect public knowledge of and compliance with newly passed ordinances? What alternative mechanisms are available to inform the public about new ordinances?

Studies describing the time course of compliance with an ordinance and tests of different strategies for improving compliance are important policy research questions. Characterising and reporting the satisfaction of smokers, non-smokers, employers, employees, government, and the public after the implementation of ordinances with different features is lacking. Such information would help to improve the design and dissemination of ordinances. A particular emphasis should be placed on the post-implementation of those groups such as restaurant associations and chambers of commerce who initially opposed the enactment of the ordinance. Descriptive studies of societal change after the passage of an ordinance can be combined with intervention studies testing different intervention strategies to consider these questions.

What is the combined impact of ordinances restricting smoking and intertwined changes in social norms on smoking behaviour is an important policy question. It is reasonable to expect that ordinances may reduce the number of cigarettes smoked each day by a smoker, may increase the number of smokers attempting to stop, and may decrease the rate of relapse in those who have stopped. What impact they have on the numbers of people who start smoking is a vital question, particularly ordinances that restrict smoking in and around schools. The characterisation of differences in norms and behaviours between different states and regions would guide the process of dissemination and provide insight into the factors that determine successful enactment and implementation. These issues may be studied by means of longitudinal population data as well as by examining cohorts before and after ordinances are implemented. The evaluation of the interaction and potential synergism of these ordinances with other modes of tobacco control, however, will require collection and analyses of data on the entire range of activities concerned with tobacco control.

The fear of substantial economic cost, particularly by restaurants, is a common argument used to counter the enactment of ordinances. Therefore, it is important to document any costs or effects on business of implementing an ordinance. Assessment of the economic impact should include all segments of the economy including business, labour, government, and other areas. This information would help shape public policy.

To track the state of current and proposed ordinances at both the local and state levels, ${ }^{16,17}$ together with the effective dissemination of these data, is a continuing need. A companion need is to track the strategies and tactics of the tobacco companies in combating these ordinances. Specific attention should be paid to the arguments and counter arguments raised against these ordinances as well as the financial contributions of the tobacco industry to legislators and other groups involved in public policy. Outreach mechanisms to advocates to identify new and evolving issues that are barriers to the development of public policy will be critical. Analyses of the tactics and contributions of the tobacco industry should go beyond simple recording of events and amounts. Important research questions involve the linkages between contributions of the tobacco industry to legislators or groups of legislators - for example, members of a legislative committee - and the enactment of defeat of legislation.

\section{WORKSITES}

The effectiveness of different policies (for example ban $v$ separate smoking and nonsmoking sections) on protecting workers from exposure to ETS, and the question of whether restrictive smoking policies cause smokers to stop or to shift jobs or work locations, are unanswered research questions. The influence of different types of smoking restrictions, particularly bans, on smoking behaviour is a critical question. The large fraction of smokers who became regular or dependent smokers at ages when they would be entering the workforce raises questions about the role of socialisation into the workforce and workplace smoking norms on the imitation of smoking and the development of nicotine dependence. The role of bans on worksite smoking in diminishing smoking uptake is important because of the employer's responsibility to provide a safe and healthy work environment. An additional research question would be the effect of different types of restrictions on the number of cigarettes smoked each day.

It is important to study the influence of different smoking policies on the rate of attempts to stop and the rate of relapse back to smoking. Although attempts at cessation are important, most attempts end in relapse; therefore the more important effect of a worksite smoking ban may be to influence relapse. This influence would be expected to be cumulative over time and is likely to require a prolonged period to be noticeable. Because work stress is commonly cited by smokers to explain relapse, however, a smoking ban at the workplace might decrease relapse by de- 
creasing smokers' opportunities to smoke at the worksite. These questions can be examined with cross sectional comparisons of the smoking behaviours of workers covered by different worksite policies.

Evaluating the special opportunities and problems of restricting smoking in restaurants and other public places through the roles and responsibilities of these locations as worksites is needed.

Follow up studies of the satisfaction of employers and employees with changes in worksite policy, particularly of those groups who opposed change would be useful to counter opposition to proposed changes in other worksites. Studies of the content and process of successful and unsuccessful policy changes would also be of benefit. Of particular interest would be analyses of the relative success of policy changes that are approached from the different emphases of health and safety at the workplace, reducing employee complaints, and as part of an overall wellness or workplace environmental quality campaign. It will also be important to define the links between policies restricting smoking in the workplace and other business practices such as the sale of cigarettes on the premises and accepting or participating in tobacco sponsorships. Further questions include documenting how often smokeless tobacco, nicorette, or non-nicotine gums are used as a substitute for cigarettes in areas where smoking is prohibited.

The economic costs and cost savings of workplace policies that restrict smoking should be documented. A major gap in our understanding of worksite smoking policies relates to small worksites. A large fraction of workforces in all industrialised countries is employed in worksites that have fewer than 50 employees, and the organisation of these worksites is different from that of a large corporation. The processes of successfully implementing workplace policies and the barriers to implementing changes in these worksites are poorly understood. It is important to develop effective strategies to reach these worksites, including approaches through franchising contracts and other organisational structures. Case studies and intervention studies are appropriate formats to consider these questions; traditional controlled intervention trials are unlikely to be practical.

PROGRESSION OF POLICY CHANGE

Adoption of policies and ordinances may be enhanced by identifying those that have been successful elsewhere, and by developing model ordinances. The rapidly changing leading edge of restrictions on smoking and the more slowly progressive average or usual level of restriction make tracking and transmission of information on the current state of the art on regulation of smoking a dynamic process. Research into the process of collection and dissemination of information, different approaches to policy evaluation, and assessment and development of model policy is promising.
There is a need for structures that define and push the existing standards for policy formation in this area. The agenda for discussion of policy change is often defined by the tobacco industry; information and activities that define a positive rather than a responsive agenda for policy change will need to be developed.

Consideration of the strengths and weaknesses of local $v$ state based policy change is needed to assess whether progress is accelerated or impeded by focusing the agenda for change on state legislators. Particular attention should be paid to the frequency and rapidity with which state legislation is strengthened once basic legislation is passed and on mechanisms to prevent pre-emption from slowing the leading edge of policy change.

The examination of successful ordinances to determine their common, transferable characteristics would strengthen the process of developing model ordinances. Similarly, studying the characteristics of communities that have successfully developed and passed ordinances would facilitate dissemination of ordinances. Equally important would be the companion study of communities in which ordinances were retracted or rescinded.

\section{Conclusion}

In summary, ample scientific evidence documents that the public must be protected from exposure to ETS. These measures will benefit both people who smoke and those who do not. Communities and businesses around the country have already recognised and acted upon this need. Research should be aimed at supporting and expediting enactment of and compliance with increasingly strong restrictions on exposure to ETS. Critical policy research questions include:

- What is the current prevalence of state and city ordinances and private sector policies, and what are current attitudes, beliefs, and behaviours regarding these ordinances and policies?

- Do smoking policies at the workplace, particularly bans, alter smoking frequency in continuing smokers, or influence cessation attempts, or rates of relapse?

- Do bans on smoking at the worksite alter uptake of smoking behaviour?

- What are the economic costs and benefits of ordinances and policies that restrict smoking? - What is the degree of satisfaction with ordinances and policies that have been implemented, particularly among those who opposed their enactment?

- What tactics have the tobacco industry used to counter the dissemination of smoke free air policies and how can these tactics be opposed? - How can model policies and information on existing policies best be used to facilitate policy change?

- What strategies facilitate and accelerate compliance with ordinances and policies that restrict smoking?

- What are the effects on smoking behaviour of policies that make schools smoke free?

- What is the relative importance of changes 
in personal attitudes, beliefs, and behaviours in promoting smoking changes in policies or ordinances and cessation among smokers?

1 US Department of Health and Human Services, Centers for Disease Control. Discomfort from environmental tobacco smoke among employees at worksites with minimal smoking restrictions - United States, 1988. MMWR 1992; 41: 351-4.

2 Repace JL, Lowrey AH. Risk assessment methodologies for passive smoking-induced lung cancer. Risk Anal 1990; 10: $27-37$.

3 Steenland $\mathrm{K}$. Passive smoking and the risk of heart disease. FAMA 1992; 267: 94-9.

3 Graham NM. The epidemiology of acute respiratory infections in children and adults: a global perspective. Epidemiol Rev 1990; 12: 149-78.

4 Weitzman M, Gortmaker S, Walker DK, et al. Maternal smoking and childhood asthma. Pediatrics 1990; 85: 505-11.

5 National Research Council, committee on passive smoking. Environmental tobacco smoke: measuring exposures and assessing health effects. Washington, DC: National Academy Press, 1986

6 US Department of Health and Human Services. The health consequences of involuntary smoking. A report of the
Surgeon General. Rockville, MD: US Department of Surgeon General. Rockville, MD: US Department of Health and Human Services, Public Health Service,
Centers for Disease Control, Center for Health Promotion and Education, Office on Smoking and Health, motion and Education, Office on Smoking and
1987. (DHHS Publication No (CDC) 87-8398.)

7 Office of Health and Environmental Assessment, Office of Research and Development, United States Environmental Protection Agency. Respiratory health effects of passive smoking, lung cancer and other disorders, Washing ton, DC: EPA, 1992. (Draft No EPA/600/690/006B.)

8 US Department of Health and Human Services. Environmental tobacco smoke in the workplace: lung cancer and other health effects. US Department of Health and Human Services, Public Health Service, Centers for
Disease Control, National Institute for Occupationa Safety and Health, 1991. (Current intelligence bulletin 54 .) 9 Taylor AE, Johnson DC, Kazemi H. Environmenta tobacco smoke and cardiovascular disease. A position paper from the Council on Cardiopulmonary and Critical Care, American Heart Association. Circulation 1992; 86 $1-4$

10 Rigotti NA, Pashos CL. No-smoking laws in the United States: an analysis of state and local actions to limit smoking in public places and workplaces. $¥ A M A 1991$; 266: 3162-7

11 Petersen LR, Helgerson SD, Gibbons CM, Calhoun CR Ciacoo KH, Pitchford KC. Employee smoking behaviour changes and attitudes following a restrictive policy on worksite smoking in a large company. Public Health Rep 1988; 103: 115-20

12 US Department of Health and Human Services. Reducing the health consequences of smoking : 25 years of progress. $A$ report of the Surgeon General. Rockville, Maryland: US Department of Health and Human Services, 1989. (DHHS publication (CDC) 89-8411.)

13 Borland R, Chapman S, Owen N, Hill D. Effects of workplace smoking bans on cigarette consumption. $A m \mathcal{F}$ Public Health 1990; 80: 178-80.

14 Sorensen G, Rigotti NA, Rosen A, Pinney J, Prible R. Effects of a worksite nonsmoking policy: evidence for increased cessation. Am $\mathcal{F}$ Public Health 1991; 81: 202-4.

15 US Department of Health and Human Services, National Cancer Institute. Strategies to control tobacco use in the United States: a blueprint for public health action in the 1990s. Washington, DC: DHHS, 1992. (NIH Publication No 92-3316.)

16 Pertschuck M, Shopland DR, eds. Major local smoking ordinances in the United States. A detailed matrix of the provisions of workplace, restaurant, and public places smoking ordinances. US Department of Health and Human Services, National Institutes of Health, National Cancer Institute, 1989. (NIH Publication No 90-479.)

17 National Cancer Institute. Major local tobacco control ordinances in the United States. US Department of Health and Human Services, National Institutes of Health, National Cancer Institute, 1992. (Smoking and Tobacco Control Monograph 3.) 